\title{
Die Bologna-Reform in der Bundesrepublik Deutschland. Erklärungen jenseits der Vorstellungen von einem hochschulpolitischen Masterplan ${ }^{1}$
}

\section{Stefan Kühl}

Wenn man die Bologna-Erklärung liest, dann ist sie auf den ersten Blick nur eine Sammlung schöner Worte und Werte. Es ist von einem „Europa des Wissens" die Rede, das eine „unerlässliche Voraussetzung" für „menschliche Entwicklung“ sei. Ein „Europäischer Hochschulraum“ könne dabei seinen „Bürgern die notwendigen Kompetenzen für die Herausforderungen des neuen Jahrtausends“ bieten. Aber nicht nur das. Ein „Europäischer Hochschulraum“ schaffe auch „ein Bewusstsein für gemeinsame Werte" und darüber hinaus ein „Gefühl der Zugehörigkeit" in einem gemeinsamen „kulturellen Raum“. Dies hätte eine zentrale Bedeutung für die „Entwicklung und Stärkung stabiler, friedlicher und demokratischer Gesellschaften".2

Bei dem Treffen der Bildungsminister Frankreichs, Deutschlands, Italiens und Großbritanniens, das ein Jahr vorher in Paris stattgefunden hatte und eine Art „Urknall“" für die Schaffung einer abgestimmten europäischen Hochschulpolitik bildete, ${ }^{3}$ hatte die dort verabschiedete Werteliste sogar noch dadurch einen besonderen Pfiff bekommen, dass sich die Bildungsminister gegen eine Reduzierung der Zusammenarbeit der europäischen Staaten auf wirtschaftliche Aspekte wandten. „Man sollte nicht vergessen“, so die Bildungsminister, dass „Europa nicht nur ein Europa des Euros, der Banken und der Wirtschaft ist“. Es müsse auch ein „Europa des Wissens“ sein. Dafür müsse ein „offener europäischer Raum für Hoch-

1 Bei diesem Artikel handelt es sich um eine auf die deutsche Situation zugespitzte Auskopplung aus meinem Buch „Der Sudoku-Effekt. Hochschulen im Teufelskreis der Bürokratie" (transcript Verlag 2012).

2 Bologna-Erklärung, Der Europäische Hochschulraum. Bologna: Gemeinsame Erklärung der Europäischen Bildungsminister 19.6.1999, S. 1f.

3 Vgl. Katrin Toens, „Die Sorbonne Deklaration. Hintergründe und Bedeutung von den Bologna Prozess", in: Die Hochschule, Jg. 16, H.2/2007, S. 37 53, hier: S. 39ff. 
schulbildung" geschaffen werden. ${ }^{4}$ In den Folgekonferenzen, auf denen sich die Bildungsminister in unterschiedlichen Abständen treffen und bei dem - nach dem im Bologna-Prozess ursprünglich das Bundesministerium anfangs alleine vorgeprescht war - das Bundesbildungsminsterium und die Kultusministerkonferenz der Ländern gemeinsam auftreten, wurde die schon beeindruckende Werteliste der Bologna-Erklärung immer weiter ergänzt. ${ }^{5}$ Studierende werden als ,gleichberechtigte Mitglieder der Hochschulgemeinschaft" gepriesen und ihre stärkere Einbindung als „kompetente, aktive und konstruktive Partner" bei der Entwicklung von Studiengängen gefordert). ${ }^{6}$ Es wird - einen in den neunziger Jahren einsetzenden internationalen Diskurs aufgreifend - betont, dass „Lebenslanges Lernen“ zentral für eine „wissensbasierte Gesellschaft“ ist. ${ }^{7}$ Im Weiterspinnen dieses Gedankens wird hervorgehoben, dass „auch außerhalb der Hochschule erworbene Kenntnisse“ - „das sogenannte informelle Lernen“ - eine wichtige Rolle spielen solle. ${ }^{8}$ Es wird betont, dass es bei der „Verwirklichung des Europäischen Hochschulraumes" - wegen Verselbstständigung des Prozesses wird das Adjektiv „europäisch“ groß geschrieben und der Begriff in den Verlautbahrungen häufig nur noch mit „EHR“ abgekürzt - darauf ankomme, Studierende auf „ein Leben als aktive Bürger in

4 Vgl. Sorbonne Erklärung, Joint Declaration on Harmonisation of the Architecture of the European Higher Education System, Paris 25. Mai 1998, S. 1.

5 Ursprünglich hat es sich um einen Zwei-Jahres-Rhythmen gehandelt. Die BolognaJubiläumskonferenz 2010 fand jedoch bereits ein Jahr nach der Konferenz in Leuven statt, und zukünftig wird an einen Drei-Jahres-Rhythmus zwischen den Konferenzen gedacht.

6 Prager Erklärung, „Auf dem Weg zum europäischen Hochschulraum" Prag: Kommuniqué des Treffens der europäischen Hochschulministerinnen und Hochschulminister am 19.5.2001, S. 3, 7; siehe auch Berliner Erklärung, „Den Europäischen Hochschulraum verwirklichen", Kommuniqué der Konferenz der europäischen Hochschulministerinnen und -minister am 19.9.2003 in Berlin.

7 Prager Erklärung a.a.O., S. 7. Interessant ist hier die Formulierung, dass Europa sich auf „eine wissensbasierte Gesellschaft und Wirtschaft“ stützt. Man kann fragen, ob in der Auffassung der Bildungsminister Wirtschaft nicht ein Teil der Gesellschaft ist. Und wie es aussehen soll, dass sich zwar Europa auf eine wissensbasierte Gesellschaft „stützt“, aber selbst nicht Teil der Gesellschaft ist. Aber auch das sind nur sprachliche Spitzfindigkeiten eines durch zu viel Begriffsarbeit verdorbenen Soziologen.

8 Bergener Erklärung, „Der Eurropäische Hochschulraum die Ziele verwirklichen", Bergen: Kommuniqué der europäischen Bildungsministerinnen und Bildungsminister, 20.5.2005, S. 4. 
einer demokratischen Gesellschaft" ${ }^{\text {“ }}$ vorzubereiten. ${ }^{9}$ Dabei spiele die Hochschulbildung durch die „Anhebung des Bildungsniveaus“ eine „bedeutende Rolle" beim „Abbau von Ungleichheit“ und „,daher" (sic) sei das „Potenzial der Einzelnen im Hinblick auf die persönliche Entwicklung“ auszuschöpfen. ${ }^{10}$ Alle Werte, die in der Bildungspolitik, so die Einschätzung von Barbara Kehm und Ulrich Teichler, ${ }^{11}$ gerade als ,gut und wichtig“ betrachtet wurden, wurden durch die einfache „Aufnahme in einen Spiegelstrich" des Communiqués heiliggesprochen.

Der Grund für diese Flucht in eine unverbindliche Werteliste ist nachvollziehbar. Die Ausgangssituationen der beteiligten Länder sind so unterschiedlich, die Interessen in den einzelnen Ländern so heterogen und die Ziele der teilnehmenden Bildungsminister so verschieden, dass es sich anbietet, sich in einer Erklärung zu Werten zu bekennen, die in ihrer Abstraktheit von allen akzeptiert werden können. ${ }^{12}$ Aber wie konnte aus dieser Sammlung vager Ideen eine Reform werden, die in nicht wenigen Ländern die Struktur des Studiums grundlegend verändert hat? Wie konnte aus einer von allen nur zu befürwortenden Werteliste eine Studienstruktur entstehen, die viele Universitäten allein schon beim verwaltungstechnischen Management ihrer Studiengänge vor ungeahnte Herausforderungen stellt?

In dem folgenden Text werden die Diffusionseffekte von einzelnen mit der Bologna-Reform assoziierten Merkmalen untersucht. Dabei wird argumentiert, dass diese Merkmale in ihren Details nicht durch eine intendierte Bildungspolitik entwickelt und dann in einem hochschulpolitischen Masterplan in der Bundesrepublik Deutschland durchgesetzt wurden, sondern das vielmehr die Bologna-Reform aus vielfältigen dezentralen „Erfindungen" an den deutschen Universitäten bestehen, die dann erst später - häufig in ihren weitestgehenden Varianten - gemeinsam durch die Bildungs-

9 Londoner Erklärung, „Auf dem Weg zum Europäischen Hochschulraum: Antworten auf die Herausforderung der Globalisierung", London: Kommuniqué der europäischen Bildungsministerinnen und Bildungsminister, 18.5.2007, S. 1.

10 Londoner Erklärung a.a.O., S. 5.

11 Barbara Kehm, Ulrich Teichler, „Mit Bachelor und Master Studiengängen und abschlüssen wohin? Eine Zwischenbilanz zum Bologna Prozess", in: Das Hochschulwesen, Jg. 54, H.2/2006, S. 57-67, hier: S. 57.

12 Es stehen nur wenige Tage zur Verfügung, um sich miteinander zu verständigen. Dass solche Communiqués intensiv vorher vorbereitet werden, ändert nichts an der Situation, dass die beauftragten Referate der Ministerien und europäischen Koordinationsgruppen kaum Möglichkeiten haben, sich das Einverständnis für die Verabredung sebr konkreter Maßnahmen einzuholen. 
ministerien des Bundesländer ratifiziert wurden. Die schnelle Diffusion wird dabei mit der Besonderheit der Kunstwährung ECTS erklärt.

\section{Zur Entstehung einer Kunstwährung}

Es fällt auf, wie in der Bologna-Erklärung und in den Folgeerklärungen der europäischen Bildungsminister fast beiläufig die Kunstwährung ECTS eingeführt wurde. In der Bologna-Erklärung wird lediglich die „Einführung eines Leistungspunktesystems" erwähnt, das die Anrechenbarkeit von an verschiedenen Universitäten erbrachten Studienleistungen einfacher machen soll. Lediglich zur Illustration wird erwähnt, dass es ein System ,ähnlich dem ECTS“ sein soll, mit dem im Rahmen der frühen Mobilitätsförderungsprogramme experimentiert wurde. ${ }^{13}$ Es wurde in der Bologna-Erklärung also offengelassen, wie das Leistungspunktesystem heißen sollte, welche Zeiteinheiten - wenn überhaupt - diesem System zugrunde gelegt werden sollten, in welchen Einheiten - in Form von Veranstaltungen oder in Modulen - diese Punkte gesammelt werden sollen, und ob dieses System zur Anrechnung von Leistungen nur jener Studierender dienen soll, die von einer Universität zu anderen wechseln, oder es auch Studierende erfassen soll, die ihr ganzes Studium an nur einer einzigen Universität zubringen. In den Folgekonferenzen wurde zwar dann schon konkreter von „ECTS“ oder einem „ECTS-kompatiblen System“ gesprochen, das auch die ,Kumulation" von Leistungspunkten ermöglicht, ${ }^{14}$ und es wurde darauf hingewiesen, dass das European Credit Transfer System auch bei der „Entwicklung“" ganzer Studiengänge eine Rolle spielt; ${ }^{15}$ insgesamt blieben die Konturen dieses zentralen Bausteins des „Europäischen Hochschulraums" in den Erklärungen der Bildungsminister vage.

Angesichts dieser nur sehr abstrakten Beschreibung dieses „Leistungspunktsystems" ist es überraschend, wie rigide dieses System sich in Deutschland darstellt. Inzwischen wird völlig selbstverständlich davon ausgegangen, dass die an anderen Universitäten erbrachten Leistungen in Zeitstunden verrechnet werden, dass alle Studierenden - nicht nur die auch an anderen Universitäten studierenden Studenten - der ECTS-Logik unterworfen werden sollen - und dass nicht nur, wie in den USA, „Kon-

13 Vgl. Bologna-Erklärung a.a.O., S. 4.

14 Vgl. Prager Erklärung a.a.O., S. 4.

15 Vgl. Berliner Erklärung a.a.O., S. 5. 
taktzeiten" in Seminaren, Vorlesungen oder Übungen, sondern alle Zeiten, die ein Student oder eine Studentin mit seinem bzw. ihrem Studium zubringt, erfasst werden. In der Bundesrepublik Deutschland wird es inzwischen als Selbstverständlichkeit angesehen, dass die Kunstwährung nur in Modulen erworben, verrechnet und getauscht werden darf und dass verpflichtend über Prüfungen sichergestellt werden muss, dass die Zeit in den Modulen nicht nur abgesessen wurde, sondern auch erfolgreich zum Wissenserwerb genutzt worden ist.

Wie konnte sich innerhalb von wenigen Jahren so deutlich der Charakter der Kunstwährung ausbilden?

\subsection{Der Stille-Post-Effekt - Wie verbreitet sich das Wissen über Bologna?}

Die sehr rigide und weit gehende Fassung der Kunstwährung ECTS konnte sich - und das mag auf den ersten Blick paradox wirken - gerade deshalb ausbilden, weil die Vorgaben der Bologna-Erklärung so vage waren. Wegen der Abstraktheit der Vorgaben der europäischen Bildungsminister, der Unklarheit darüber, welche Richtlinien überhaupt zu beachten sind, und wegen der vielfältigen Interpretationsmöglichkeiten nebulöser Aussagen herrschte bei den deutschen Studiengangsgestaltern große Unsicherheit darüber, was möglich ist und was nicht. Man wusste gerade in den ersten Jahren nach der Verabschiedung der Bologna-Erklärung einfach nicht, ob man die in ECTS-Punkten ausgedrückten Veranstaltungen und Prüfungen in Module zusammenfassen sollte oder nicht, wie groß oder klein die Module sein durften, wie genau die Modulbeschreibungen sein sollten und wie die Verrechnung mit ECTS-Punkten überhaupt aussehen sollte.

So bildeten sich in Deutschland auf nationalen, regionalen und lokalen Ebenen Gerüchteküchen, was „durchgehen wird" und „was nicht". Schon auf der Ebene der Wissenschafts- und Bildungsministerien der Länder im Gegensatz zur EU die einzige Ebene, auf der verbindliche Richtlinien für die Bildungspolitik erlassen werden konnten - wurde spekuliert, welche Form der „Kunstwährung“ sich europaweit durchsetzen wird. Auf der Ebene der Leitung der deutschen Universitäten wurde dann spekuliert, wie die häufig auch eher nebulösen Rahmenrichtlinien der nationalen und regionalen Bildungsministerien wohl von den für die Genehmigung zuständigen Akkreditierungs- und Genehmigungsstellen - und darauf aufbauend 
dann von den Begutachtern vor Ort - interpretiert werden würden. In den Fakultäten, Fachbereichen und Instituten, also auf der Ebene, die letztlich für die Entwicklung der Studiengänge zuständig ist, konnte dann wiederum spekuliert werden, welche der häufig sich widersprechenden Richtlinieninterpretationen sich auf Universitätsebene durchsetzen werden und wie viel Spielraum man innerhalb dieser Richtlinien hat.

In der Organisationsforschung wird diese Veränderung von Informationen über mehrere „Interpretationsstellen“ als „Stille-Post-Effekt" bezeichnet. ${ }^{16}$ Häufig werden die Informationen auf dem Weg durch verschiedene Organisationen immer wieder geändert und modifiziert. ${ }^{17}$ Jede Stelle, durch die die Information läuft, ergänzt, verändert oder kürzt, so dass die Information, die auf der operativen Ebene oder an der Spitze der Organisation ankommt, häufig wenig mit der ursprünglichen Information zu tun hat. Man braucht sich nur mal mit einer Sachbearbeiterin zu unterhalten, die durch Zufall einen Vermerk in die Hand bekommt, für den sie ursprünglich den ersten Entwurf geschrieben hat und den man aufgrund der Veränderungen auf den nächsthöheren Hierarchiestufen kaum noch wiedererkennt. ${ }^{18}$

16 Die Idee, das Konzept des für Organisationen allgemein bereits beschriebenen „Stille-Post-Effekts“ auf den Bologna-Prozess zu übertragen, stammt von Bernd Kleimann. Soweit ich weiß, hat er diesen Gedanken noch nicht in einer Publikation dargelegt.

17 Vgl. Niklas Luhmann, Politische Soziologie, Frankfurt a.M., 2010, S. 202.

18 Vgl. Stefan Kühl, Organisationen. Eine sehr kurze Einführung, Wiesbaden 2011, S. 74ff. Nur begrenzt übertreibend ist der unter Soldaten verbreitete Witz über den „Stille-Post-Effekt" in der Armee. „Der Oberst zum Oberstleutnant: Morgen früh um neun ist eine Sonnenfinsternis, etwas, was nicht alle Tage passiert. Die Männer sollen im Arbeitsanzug auf der Straße antreten, und ich werde ihnen das seltene Schauspiel erklären. Falls es regnet, werden wir nichts sehen. Dann sollen die Leute in die Sporthalle gehen." Der Oberstleutnant zum Hauptmann: „Befehl von Herrn Oberst: Morgen um neun ist eine Sonnenfinsternis. Wenn es regnet, kann man sie von der Straße aus sehen. Ansonsten findet sie im Arbeitsanzug in der Sporthalle statt, was nicht alle Tage passiert.“ Der Hauptmann zum Oberleutnant: „Morgen früh um neun alle Leute im Arbeitsanzug zu einer Sonnenfinsternis in die Sporthalle. Falls es regnet, wird der Herr Oberst Befehl geben, auf der Straße anzutreten, was nicht alle Tage passiert.“ Der Oberleutnant zum Leutnant: „Morgen früh um neun wird der Herr Oberst im Arbeitsanzug in der Sporthalle die Sonne verfinstern, was nicht alle Tage passiert. Wenn es regnet, dann antreten auf der Straße.“ Der Leutnant zum Fähnrich: „Morgen um neun Sonnenfinsternis des Herrn Oberst im Arbeitsanzug. Wenn es in der Sporthalle regnet, was nicht alle Tage passiert, antreten auf der Straße." Fähnrich an alle Kompanien: „Wenn es 
Auffällig ist jedoch, dass der Stille-Post-Effekt der Bologna-Reform den vermeintlichen Spielraum für die deutschen Bundländerländer, für die deutschen Universitäten und für deren Fakultäten, Fachbereiche und Institute immer weiter eingeschränkt hat. Haucke Brunckhorst ${ }^{19}$ stellt fest, dass schon auf der nationalen Ebene die ,in Wahrheit völlig unverbindlichen Empfehlungen einer Versammlung diverser Minister und Staatssekretäre" so präsentiert wurden, als wenn ihnen „unbedingt Folge zu leisten wäre“, frei nach dem Motto „Höhere Wesen befahlen....".20 Auf der Ebene der deutschen Universitäten - und weitergehend auf der Ebene der Fakultäten, Fachbereiche und Institute - wird dieser Prozess fortgeführt, wenn sehr enge Regelungen mit vermeintlichen Vorgaben, Richtlinien und Präzedenzentscheidungen von den in der Bundesrepublik neu geschaffenen Akkreditierungsagenturen begründet werden, frei nach dem Motto „Interessante Idee, aber das bekommen wir bei der Akkreditierung nie durch". ${ }^{21}$

morgen regnet, wird der Oberst anscheinend in der Sporthalle von der Sonne verfinstert. $\mathrm{Zu}$ dumm, dass so etwas nicht alle Tage passiert." Es fehlt meines Wissens in Armeen noch ein Witz, der die Verzerrung von Informationen von unten nach oben aufgreift (vgl. für einen ansonsten sehr guten Überblick über Witze in Organisationen Oswald Neuberger, Was ist denn da so komisch, Weinheim, Basel 1988.).

19 Hauke Brunckhorst, „So wird Sachzwang gebaut", in: TAZ. die tageszeitung, 12.8.2006.

20 Vgl. auch Robert Pfaller, „Der Kampf gegen die Fortentwicklung der Universität zur repressiven Attrappe" in: Johanna Charlotte Horst (Hrsg.), Unbedingte Universitäten. Was passiert? Stellungnahmen zur Lage der Universität, Zürich 2010, S. 51-54, hier S. 54.In der politikwissenschaftlichen Governance-Forschung, die jedenfalls in einigen ihrer prominenten Spielarten hinter jeder Entscheidung Intentionen vermuten (und nicht wie bei den Systemtheoretikern: Zurechnungen), wird von dem Konzept einer „Neuen Staatsraison“ (vgl. zum Beispiel Kerstin Martens, Klaus Dieter Wolf, "Paradoxien der Neuen Staatsräson. Die Internationalisierung der Bildungspolitik in der EU und der OECD", in: Zeitschrift für Internationale Beziehungen, Jg. 13, H.2/2006, S. 145-176.) gesprochen und geschrieben. Die Erklärungen internationaler Ministerzusammenkünfte und Deklarationen internationaler Meta-Organisationen, deren Mitglieder die Nationalstaaten sind, werden von den nationalen Regierungen dafür genutzt, um politische Widerstände zu brechen. Die internationalen Organisationen und die internationalen Zusammenkünfte von Ministern übernehmen, so Jens Maeße dabei die „Rolle von Stichwortgebern“ für die nationalen Gesetzgeber, vgl. Jens Maeße, „Die vielen Stimmen des Bologna Prozesses. Zur diskursiven Logik eines bildungspolitischen Programms", Bielefeld 2010.

21 Es kursieren Geschichten von Akkreditierungen, in denen Professoren, die im Namen einer der Akkreditierungsagenturen einen neuen Studiengang prüften, die 
Diese Tendenz einer immer engeren Auslegung der Informationen im Laufe der Weitergabe in der "Stille-Post-Kette“ lässt sich an verschiedenen zentralen Aspekten des Reformprozesses belegen. Anfangs war bei den Treffen der Wissenschafts- und Bildungsminister der Bundesländer noch nicht einmal die Rede davon, dass Leistungspunkte nur in der Container-Form von Modulen erworben, verrechnet und umgetauscht werden müssen, dann wurde irgendwann in der Praxis der Studiengangsentwicklung bei den meisten Unis davon ausgegangen, dass jede einzelne Veranstaltung, jede einzelne Prüfung Teil eines solchen Moduls sein muss, und später kursierten Gerüchte, dass es nicht möglich sein dürfte, dass ein Modul aus einer einzigen Veranstaltung besteht. Ähnlich verhielt es sich mit Auffassungen über Prüfungen. Weil Module als Konzept von den Bildungs- und Wissenschaftsministern auf der Bologna-Konferenz noch gar nicht erwähnt wurden, gab es natürlich auch keine Vorstellung darüber, ob und wie Module abgeprüft werden müssten. So bildeten sich dann auf der Ebene der deutschen Universitäten Gerüchte aus, dass jedes Modul notwendigerweise durch eine Prüfung abgeschlossen werden müsste, bis dann bei der Studiengangsplanung immer wieder auch Stimmen zu hören waren, dass solche Prüfungen zu benoten seien und diese Noten verpflichtend in die Endnote eines Studiums eingehen müssten. Ganz ähnlich dann auch bei der Formulierung von Lernzielen: Anfangs gab es lediglich unter Studiengangsplanern kursierende Informationen, dass in einem Modul ,thematisch zusammenhängende Veranstaltungen und Prüfungen" zusammengefasst werden sollten. Es kamen Gerüchte auf, dass Module nur dann durch die Universitätsleitung, durch die Akkreditierungsbehörde, durch das Bildungsministerium, durch die europäischen Bildungsminister oder in der interessantesten Form des Gerüchts - durch die Staatschefs der Europäischen Union genehmigt werden würden, wenn das Lernziel für jedes Modul ausführlich und trennscharf zu den anderen Modulen beschrieben werden würde, bis sich dann in einigen Universitäten die Interpretati-

Studiengangsentwickler darauf hinwiesen, dass ihre geplanten kleingliedrigen Modulen mit fünf Leistungspunkten, die jeweils nur mit zwei Veranstaltung pro Jahr bedient werden können, den Studierenden faktisch jede Form Wahlmöglichkeit nehmen würden. Die Studiengangsentwickler hielten den mit der Prüfung beauftragten Professoren entgegen, dass große „Sammelmodule“ bei der Akkreditierung nicht durchgehen würden. Die Ironie, die darin liegt, dass sie dieses Argument einer vermeintlichen Entscheidungspraxis von Akkreditierungsagenturen gerade den Professoren gegenüber vorbrachten, die im Namen der Akkreditierungsagentur den Studiengang begutachteten, ist in der Sitzung nicht aufgefallen. 
on durchsetzte, dass Studierende selbst bei permanent wechselnden inhaltlichen Veranstaltungen in einem Modul jedes Modul nur ein einziges Mal studieren dürften, weil man ja durch das Belegen eines aus zwei Seminaren bestehenden Moduls „Englische Literatur", „Soziologische Theorie“ oder „Buddhismus" alles gelernt haben muss, was man zu diesem Thema entsprechend der definierten Lernziele wissen muss. ${ }^{22}$

Aber - diese Frage liegt auf der Hand - weswegen führte der „StillePost-Effekt " nicht zu einer großen Interpretationsfreiheit auf der Ebene der Institute, Fachbereiche und Fakultäten, sondern im Gegenteil in den meisten Fällen dazu, dass sich Interpretationen durchsetzten, die Handlungsräume als eher gering erschienen ließen?

\subsection{Deutsche Universitäten im Wettbewerb um die „Bologna-Reinheit “}

Nach der Verkündigung der Bologna-Erklärung gab es kaum Vorstellungen darüber, wie diese hehren Ziele vor Ort umgesetzt werden sollen. ${ }^{23}$ Angesichts dieser Unklarheit hatten Universitäten eigentlich nur zwei Möglichkeiten: Sie konnten abwarten, bis die vagen Formulierungen der europäischen Bildungsminister durch ihr Landesministerium in verbindliche Richtlinien umgesetzt werden, oder sie konnten anfangen, im Rahmen von einigen nur sehr vagen Vorgaben der Bildungsministerien zu experimentieren.

Interessant ist, dass ,ausgehend von den Schlagworten der Bolognatexte" sich viele deutsche Universitäten „selbstständig auf die Suche nach möglichen Bedeutungen" begeben haben. Universitäten fingen an, Umsetzungsmöglichkeiten für die Proklamationen der Bologna-Reform zu suchen, Modell-Studiengänge $\mathrm{zu}$ entwickeln und eigene universitätsweite

22 Auch hier fehlt es an ausführlichen Mikrostudien, wie sich in den einzelnen Ländern bestimmte Interpretationen ausgebildet haben. Es ist nur zu hoffen, dass es statt der immer wieder gleichen Aufzählung der groben Ziele der verschiedenen Konferenzen der Bildungsminister in absehbarer Zeit solche Detailstudien über die Rigidisierungen (und auch Auflockerungen) einzelner Elemente der Hochschulreform geben wird:

23 Vgl. Lutz R Reuter, "Zur Transformation der nationalen Hochschulsysteme durch die Europäische Union: Der „Bologna Prozess". Ziele, Entwicklungen, Kritik”, in: Hans-Werner Fuchs, Lutz R. Reuter, Internationalisierung der Hochschulsysteme: der Bologna-Prozess und das Hochschulwesen der USA, Hamburger Beiträge zur Erziehungs und Sozialwissenschaft, H. 6/2003, S. 9-26; Maeße a.a.O., S. 22. 
Richtlinien für die Einrichtung von zweigliedrigen Studiengängen und die Verrechnung von ECTS-Punkten zu erlassen. Jens Maeße spricht hier für die Bundesrepublik Deutschland von einem „kreativitätsstiftenden Charakter" der Bologna-Reform, die "streng genommen keine Reform", sondern eher „eine Aufforderung zur Reform“ "gewesen ist. ${ }^{24}$

Aber weswegen ließen sich die deutschen Universitäten überhaupt auf solche Experimente ein? Weswegen warteten viele Universitäten nicht ab, welche übergreifenden Richtlinien sich ausbilden, welche Fehler andere machen, um dann möglichst gleich im ersten Wurf ein konsistentes und auf der Erfahrung anderer aufbauendes Studiengangskonzept umzusetzen? Weswegen bildete sich schon kurz nach der Verabschiedung der Erklärung der Bildungsminister ein Wettbewerb der deutschen Universitäten um die besten „Bolognakonformen Studiengänge“ ${ }^{\text {25 }}$ aus?

Auf der internationalen Ebene schien die Bundesrepublik Deutschland sich relativ schnell an einem internationalen "Prestigekampf" beteiligt zu haben, in dem es darum ging, wer am „schnellsten“ und am „besten“ die Strategie umgesetzt hat. ${ }^{26}$ Vor den Folgekonferenzen wurden Fortschrittsberichte aus jedem Bologna-Land eingefordert, und es wurde verglichen, wie viel Prozent der Studiengänge auf eine Bachelor- und Masterstruktur umgestellt wurden, wie umfassend die Berechnung jeder Arbeitsstunde von Studierenden in ECTS-Stunden umgesetzt wurde und welche Modelle der Modularisierung entwickelt wurden. Länder, die auf diesen Konferenzen keine Erfolge vorzuweisen hatten, drohten schnell das Image eines "Nachhinkers" zu bekommen.

Aus diesem Grund drängten die Landesministerien in der Bundesrepublik Deutschland darauf, dass die Universitäten möglichst schnell „Bolognakonforme Studiengänge" einführen, und nutzten dazu ein neues Steuerungsinstrument gegenüber den Universitäten. Seit den 1990er Jahren schließen immer mehr deutsche Universitäten mit den Landesministerien Leistungsvereinbarungen ab. Dabei werden nicht nur quantitative Vorgaben wie Studierendenzahlen, Abschlussquoten oder Drittmittelquoten vereinbart, sondern auch Zeitpläne für die Umstellung von Forschungs- oder

24 Maeße a.a.O., S. 132.

25 Alice Peters Burns, Margret Schermutzki, „Die Umstellung auf kompetenz-basierte Learning Outcome Orientierung an der Fachhochschule Aachen", in: Hochschulrektorenkonferenz Bologna Zentrum (Hrsg.), Bologna in der Praxis. Erfahrungen aus den Hochschulen, Gütersloh 2008, S. 99-119, hier S. 102.

26 Maeße a.a.O., S. 47. 
Studienstrukturen. Für deutschen Universitäten bestand damit nicht nur die Notwendigkeit, ihre in den Leistungsvereinbarungen verabredeten Studiengangsumstellungen einzuhalten, sondern es entstand auch die Möglichkeit, sich gegenüber den Ministerien durch eine besonders schnelle Einführung als „Planübererfüller“ zu profilieren. ${ }^{27}$

Dieser Prozess wurde durch die Europäische Union, die nationalen Bildungsministerien und die regionalen Bildungsverwaltungen durch das Auflegen von Förderprogrammen befördert. Es wurden Projekte mit so wohlklingenden Namen wie "Tuning Educational Structure in Europe" aufgelegt, durch die Universitäten sich eine Anschubfinanzierung für das Experimentieren mit „Bolognakonformen Studiengängen“ sichern konnten. Die Methode der Umsetzung über öffentlich bezuschusste Experimente, Evaluation der Erfahrungen, Austausch auf Konferenzen und Zusammenführung in Projekterfolgsberichten wird in der Politikwissenschaft als "Offene Koordinationsmethode“ - „Open Methode of Coordination" - bezeichnet. $^{28}$

Diese Umstellung einzelner Studiengänge im Rahmen von Reformprozessen produzierte dann eine Sogwirkung für die Umstellung aller anderen Studiengänge einer Universität. Wenn ein Fachbereich einen einzelnen Studiengang beispielsweise für Politikwissenschaft umstellte, dann war es begründungspflichtig, weswegen nicht gleichzeitig auch der Studiengang für Soziologie auf eine Bachelorstruktur umgestellt wird, weil für den Studiengang Politikwissenschaft ja sowieso die Grundstrukturen des Nebenfaches Soziologie in ECTS-Punkten ausgedrückt werden müsse. Wenn eine Universität die Lehrerausbildung erst einmal nur für Englisch und Biologie auf Bachelor und Master umstellen will, können sich andere Lehramtsfächer wie Physik, Deutsch oder Geschichte dem dann nur noch

27 Hierzu fehlen meines Wissens nach noch empirische Forschungen. Aber erste empirische Betrachtungen sprechen dafür, dass Universitäten in den Regionen (wie beispielsweise Bayern), deren Ministerien in ihren Leistungsvereinbarungen lange Zeit nicht auf die Umsetzung gedrängt hatten, die Bologna-Studiengänge deutlich später umgesetzt haben als Universitäten in den Ländern, die diese Umsetzung sehr früh in den Leistungsvereinbarungen vorsahen.

28 Siehe z.B. Amélia Amaral, Alberto Veiga, "The Open Method of Coordination and the Implementation of the Bologna Process", in: Tertiary Education Management, Jg. 12, H.4/2006, S. 283-295, hier S. 283ff. Oder zur Anwendung der Methode auf die Bolognareform Thomas Walter, Der Bologna Prozess. Ein Wendepunkt europäischer Hochschulpolitik?, Wiesbaden 2006. 
schwer entziehen, weil ein Lehramtsstudium an einer Universität ja „aus einem Guss" angeboten werden muss.

\subsection{Die Ratifizierung des Erprobten}

Angesichts dieser Entwicklung der Hochschulreform „,von unten“ ist es erst einmal plausibel, wenn Jens Maeße feststellt, dass der Prozess der Hochschulreformen weder in Bologna noch an den Orten der Folgekonferenzen in Prag, Berlin, Bergen, London, Leuven, Wien oder Budapest gemacht wurde, sondern „überall dort, wo sich Wissenschaftspolitiker, Hochschulangehörige und Bildungsexperten auf die Suche nach möglichen Bedeutungen und den naheliegenden Umsetzungsmöglichkeiten des Bologna-Prozesses gemacht haben" ${ }^{29}$ Auffällig ist dabei auch, wie zurückhaltend - bewusst oder unbewusst - anfangs die Bildungspolitiker in Berlin oder in den Ländern damit gewesen sind, diese ,Suche nach Bedeutungen und möglichen Umsetzungsmöglichkeiten" durch zu verbindliche Rahmenrichtlinien zu beeinflussen. Man fühlte sich ein bisschen an die Mao Zedongs kurzzeitige Aufforderung an die chinesische Bevölkerung „Lasst hundert Blumen blühen, lasst hundert Schulen miteinander wetteifern" erinnert, mit dem er einen Wettstreit, um den richtigen Weg zu einer besseren Gesellschaft initiieren wollte. ${ }^{30}$

Aber im Laufe des Prozesses wurde durch das hohe Maß an Heterogenität bei den Interpretationen vor Ort der Druck zu einer Vereinheitlichung fast automatisch immer größer. Schließlich war das Ziel des Bologna-Prozesses ja nicht ein Flickenteppich von lokalen Lösungen, sondern ein System, das die Vergleichbarkeit von Studienleistungen landes-, wenn nicht sogar europaweit sicherstellte. Mit Verweis auf die Notwendigkeit einheitlicher Interpretationen der Kunstwährung ECTS wenigstens innerhalb der Bundesrepublik Deutschland verabschiedeten die Bildungspolitiker dann deswegen zunehmend für die Universitäten rechtlich verbindliche Verordnungen. Bei den Gesetzen oder Strukturvorgaben wurden dann - siehe der

29 Maeße a.a.O., S 132.

30 Vgl. Ulrich Teichler, Hochschulstrukturen im Umbruch. Eine Bilanz der Reformdynamik seit vier Jahrzehnten, Frankfurt a.M., New York 2005, S. 307; siehe ausführlich zur Hundert-Blumen-Bewegung in China Roderick MacFarquhar, The Origins of the Cultural Revolution: Contradictions Among the People, 19561957. New York 1973. 
internationale Wettstreit um eine Vorreiterrolle im Bologna-Prozess - häufig in vielen Bundesländern die rigideren Deutungen und die am weitesten gehenden Umsetzungsversuche durch Verordnungen ratifiziert.

In der Soziologie wird ein solches, rechtlich schließlich verbindliches Vorschreiben von rigiden Strukturen, die sich eigentlich nur aufgrund von häufig sehr vagen oder gar falschen Annahmen über mögliche zukünftige Entscheidungen der höheren Instanzen ausgebildet haben, als „,selbsterfüllende Prophezeiung" - self-fulfilling prophecy - charakterisiert. Mit diesem Begriff bezeichnet Robert Merton ${ }^{31}$ das Phänomen, dass falsche oder unzureichende Vorhersagen, Interpretationen und Urteile einen sozialen Prozess so prägen können, dass dadurch, dass sich alle Akteure an diese ursprünglich falschen oder unzureichenden Annahmen anpassen, diese sich letztlich als richtig verfestigen. Man kennt den Effekt von Banken, die eigentlich über eine gute Liquidität verfügen, durch falsche Gerüchte über ihre mögliche Zahlungsunfähigkeit jedoch gezwungen werden, den sich nur aufgrund Gerüchte an den Schaltern drängenden Kunden ihre Einlagen auszuzahlen und erst wegen dieses rapiden Mittelabflusses in die Insolvenz gezwungen werden. ${ }^{32}$ Durch die Psychologie ist der sogenannte „Pygmalion-Effekt“" nachgewiesen, wonach die Leistungserwartungen, die Lehrenden ihren Schülern und Studenten entgegenbringen, deren faktische Leistungsfähigkeit bestimmen. Es ist experimentell nachgewiesen worden, dass, wenn man Lehrer von der Intelligenz eines Schülers überzeugt, der in Wirklichkeit lediglich per Zufall ausgewählt wurde, die Lehrer von diesem mehr erwarten und am Ende dieser Schüler deswegen auch derjenige mit den höchsten Leistungssteigerungen ist. ${ }^{33}$ Und seit dem Bologna-Prozess können wir auch beobachten, wie häufig nur auf Spekulationen basierende restriktive Interpretationen und Annahmen über die Entscheidung von staatlichen Genehmigungsbehörden, Akkreditierungsagenturen oder Universitätsgremien dazu führen, dass diese ursprünglich überzogenen rigiden Interpretationen irgendwann in Gesetze und Verordnungen gegossen werden.

31 Robert K. Merton, Soziologische Theorie und soziale Struktur. Berlin, New York 1995 , S. $401 \mathrm{ff}$.

32 Vgl. Merton a.a.O., S. $399 \mathrm{ff}$.

33 Robert Rosenthal, Lenore Jacobson, Pygmalion im Unterricht, Weinheim 1971. 


\section{Die Diffusion einer Kunstwährung}

Eigentlich hätte man davon ausgehen können, dass der von den europäischen Bildungsministern in Bologna angestoßene Prozess sicher zum Scheitern verurteilt ist. Die Bologna-Erklärung hat keinerlei rechtliche Verbindlichkeit für die Unterzeichnerstaaten, weil in den Grundlagenverträgen der Europäischen Union eindeutig geregelt ist, dass die Bildungspolitik einzig und allein in die Kompetenz der jeweiligen Nationalstaaten fällt und Vereinbarungen auf EU-Ebene zur Bildungspolitik keine Verbindlichkeiten für die Unterzeichnenden nach sich ziehen. Alle früheren Initiativen zur Hochschulpolitik der Europäischen Kommission wie z. B. ein Memorandum zum Europäischen Hochschulraum wurden deswegen von den Regierungen der europäischen Staaten argwöhnisch beäugt und dann faktisch ignoriert. ${ }^{34}$ Umso erstaunlicher ist, mit welcher Geschwindigkeit, sich der Bologna-Prozess durchsetzte - eine Diffusion, die sich, so die These, nur durch die Wirkweise der Kunstwährung ECTS erklären lässt.

\subsection{Die räumliche Ausbreitung einer Kunstwährung}

Als Erstes sticht ins Auge, wie schnell sich der Bologna-Prozess räumlich ausgebreitet hat. Während in der Vorbereitung zur Bologna-Konferenz nur die Bildungsminister Deutschlands, Italiens, Englands und Frankreichs in einer Erklärung die Einführung einer gemeinsamen Kunstwährung anregten, waren es bei der Bologna-Konferenz bereits dreißig europäische Staaten. Nur zehn Jahre nach der Verabschiedung waren es dann schon sechsundvierzig Staaten, die dem Bologna-Prozess beigetreten waren. Selbst Staaten wie Moldawien, Russland oder Kasachstan, die normalerweise nicht unbedingt zu Kernstaaten der Europäischen Union gerechnet werden, bekennen sich inzwischen zu den Prinzipien eines gemeinsamen europäischen Hochschulraums. Und es ist jetzt schon zu beobachten, dass auch Staaten in Asien, Afrika, Amerika oder Australien die Entwicklungen in Europa mit Interesse zur Kenntnis nehmen und als Beobachter an den Konferenzen der Bildungsminister teilnehmen.

34 Vgl. zu dem Aspekt des erwarteten Scheiterns ausführlich Maeße a.a.O., S. 19. 
Dabei überrascht weniger die Bereitschaft, sich in Erklärungen zu so hehren Werten wie „Förderung der internationalen Mobilität“, „Sicherung von Qualität in der Bildung“ oder „Zugang zu Bildung unabhängig von sozialer Herkunft" zu bekennen. Schon die Erfahrungen der UNESCO, der UN-Organisation für Erziehung, Wissenschaft und Kultur, zeigt seit ihrer Gründung im Jahr 1945, dass es (fast) allen ungefähr zweihundert Staaten der Weltgesellschaft leicht gefallen ist, sich zu abstrakt formulierten Werten wie Wissenschaftsförderung, Bildungsgerechtigkeit, Gesundheitserziehung, Geschlechtergleichstellung oder Kulturpflege zu bekennen. Den Beitritt zu einem lockeren Bündnis, in dem vorrangig das Bekenntnis zu allgemein akzeptierten Werten gepflegt wird, ist also billig und überrascht deswegen nicht. Überraschender ist in Bezug auf den Bologna-Prozess also vielmehr, wie bereitwillig die Staaten - neben der Zweigliedrigkeit eines berufsqualifizierenden Basisstudiums und eines darauf aufbauenden Masterstudiums - die Kunstwährung ECTS übernommen haben.

Diese Bereitwilligkeit zur Übernahme einer Kunstwährung ist darauf zurückzuführen, dass in den betreffenden Staaten in der Regel keine eigene Zeitwährung zur Berechnung der Arbeitslast zur Verfügung stand. Es bestand in diesen Staaten bisher keine Verrechnungseinheit, mit der die Zeit der Studierenden für die Vor- und Nachbereitung von Veranstaltungen, für das Absolvieren von Prüfungsleistungen oder das Verfertigen von Hausarbeiten gemessen wurde, so dass es keine Konkurrenz zu der neuen Kunstwährung gab. Der Expansionsprozess der Kunstwährung ist vergleichbar zum Vordringen einer Geldwährung in eine Region, in der bisher lediglich Gütertausch bekannt war. Die Übernahme der neuen Währung verspricht nicht nur, den Vergleich und die Verrechnung mit neuen Tauschpartnern möglich zu machen, sondern auch den Vergleich und die Verrechnung im Inneren zu erleichtern, ohne dass dafür bestehende Berechnungsformen gänzlich aufgegeben werden müssen.

Diese räumliche Expansion der Kunstwährung stößt erst dann an Grenzen, wenn in einem Land bereits eine andere, gut eingeführte Bildungswährung vorhanden ist. Schließlich erscheint es nicht einleuchtend, weswegen man eine Währung übernehmen soll, wenn es doch bereits eine eigene Währung gibt, mit der das Gleiche - vielleicht nur auf andere Art und Weise - berechnet werden muss. Wie bereits die Schwierigkeiten der Verrechnungen zwischen an europäischen und US-amerikanischen Universitäten erbrachten Leistungen zeigen, werden dann eigene Modelle zur Verrechnung der Studienleistungen etabliert, die häufig noch nicht einmal 
Bezug nehmen auf die in den beiden Regionen herrschenden Verrechnungseinheiten.

\subsection{Die soziale Ausweitung einer Kunstwährung}

Es fällt uns fast nicht mehr auf, dass die vermeintliche Arbeitsbelastung für jeden Studenten, jede Studentin in einem Bologna-Studiengang in der Kunstwährung ECTS berechnet wird. Dabei ging es - wie gezeigt - anfangs lediglich um die „Einführung eines Leistungspunktesystems“, um Studierenden, die an mehreren Universitäten studieren, die Anrechenbarkeit von Studienleistungen zu ermöglichen. Erst im Laufe des Prozesses bildete sich das Prinzip heraus, das Kreditpunktesystem nicht nur zum Transfer von Leistungen zwischen verschiedenen Universitäten zu benutzen, sondern es auch verpflichtend für die Studierenden vorzusehen, die $i h r$ gesamtes Studium an einer einzigen Universität verbringen.

Diese soziale Ausweitung der Kunstwährung überrascht, weil vor und nach dem Anstoßen des Bologna-Prozesses nur eine geringe Anzahl von Studierenden überhaupt während eines Studiums zwischen Universitäten wechselt. Kritische Beobachter vergleichen deswegen die verbindliche Einführung der ECTS-Punkte, die schon bei ihrer Erprobung für die Mobilitätsprogramme der EU in den 1990er Jahren nicht gerade handlich gewesen sind, mit einem Gips, den man nicht nur Studierenden mit einem gebrochenen Bein verordnet, sondern vorsorglich allen.

Aber selbstverständlich wird die Bezeichnung von ECTS-Punkten als ein Gips für Gesunde von den wenigsten Verfechtern der Bologna-Reform geteilt. Vielmehr wurden zusätzliche Ziele entdeckt, die mit dem eigentlich nur für das Ziel „Mobilitätsförderung“ gedachten Mittel ECTS-Punkte erreicht werden können. Plötzlich wurde das Argument gebracht, dass die ECTS-Punkte ja nicht nur für die Verrechnung der Mobilität zwischen Universitäten verschiedener Staaten genutzt werden können, sondern auch für die Verrechnung verschiedener Veranstaltungen zwischen verschiedenen Instituten einer Universität. Schnell war dann auch die Rede davon, dass mit ECTS-Punkten der Zeitaufwand für einen Studiengang genauer bestimmt werden kann und durch eine Berechnung des gesamten Studiengangs in dieser Zeitwährung sowohl eine Unter- als auch Überforderung von Studierenden verhindert werden kann.

Diese „Erfindung“ von neuen Zielen für ein ursprünglich für ein ganz anderes Ziel eingeführtes Mittel ist in der Organisationsforschung sehr gut 
untersucht. Hat man, um ein Beispiel aus dem Bereich der Wirtschaft zu bringen, als Weltbank nach dem Zweiten Weltkrieg Know-how für das Management von Projekten zum Wiederaufbau in Europa entwickelt, bietet es sich an, dieses Wissen auch für die Durchführung und Finanzierung von Projekten in sogenannten ,unterentwickelten“ Ländern in Asien, Afrika und Lateinamerika zu nutzen. ${ }^{35}$

Normalerweise wird aber in der Organisationsforschung davon ausgegangen, dass sich vielfältige Hürden auftun, wenn Mittel, die für ein bestimmtes Ziel entwickelt wurden, für neue Ziele eingesetzt werden sollen. Im Fall des Bologna-Prozesses war diese Ausweitung des Mitteleinsatzes vergleichsweise unproblematisch, weil die Leistungsanforderungen sowieso in einer numerischen Form gefasst sind. Hat man erst einmal ein auf Zeitstunden basierendes Transfersystem etabliert, dann ist es ein vergleichsweise geringer Aufwand, dieses System auch für die Akkumulation von Leistungen im Rahmen eines Studienganges zu nutzen. Wenn man sowieso als Maßnahme der Mobilitätsförderung alle an einer Universität angebotenen Veranstaltungen in eine Kunstwährung umrechnen muss, dann kann man - so die Logik - auch gleich die gesamte Planung von Studiengängen auf dieser Kunstwährung aufbauen.

\subsection{Die sachliche Ausdehnung einer Kunstwährung}

Ursprünglich war die Kunstwährung ECTS nur zur Berechnung des Arbeitsaufwandes für einzelne Veranstaltungen und Prüfungen gedacht. Hat man aber erst einmal den vermeintlichen Arbeitsaufwand von Studierenden für jede Veranstaltung und jede Prüfung bestimmt, kann man diese Zahl nahezu beliebig mit anderen Zahlen kombinieren.

Diese Aufladung der ECTS-Punkte durch die Kombination mit anderen Zahlen betrifft erst einmal vorrangig die Studierenden. Ein Feld, in dem die Kunstwährung ECTS bereits vielfach mit anderen Zahlen kombiniert wird, ist die Notengebung. Weil ECTS-Punkte nur den durchschnittlichen Arbeitsaufwand von Studierenden messen, müssen getrennt davon die Noten für Arbeitsleistungen erhoben werden. In vielen Universitäten werden die Noten bereits schematisch nach der Anzahl der Leistungspunkte für

35 Vgl. Stefanie Hanke, „Weiß die Weltbank, was sie tut? Über den Umgang mit Unsicherheit in einer Organisation der Entwicklungsfinanzierung", in: Soziale Systeme, Jg. 2, H.2/1996, S. 331-360. 
ein Arbeitspaket gewichtet. Je mehr Leistungspunkte für ein Modul vergeben werden, desto wichtiger ist die Note für dieses Modul für die Endnote. Aber Universitäten experimentieren schon damit, die Zulassung zu weiterführenden Studiengängen davon abhängig zu machen, wie viele Leistungspunkte während des Bachelorstudiums in den für den Masterstudiengang relevanten Modulen erworben werden. Eine Zulassung zu einem Studiengang „Politische Kommunikation“ erhält man dann eben nur, wenn man während des Bachelorstudiums mindestens 60 Leistungspunkte in Politikwissenschaft und 60 Leistungspunkte in Kommunikationswissenschaft erworben hat. Aber auch ist jetzt schon - basierend auf Erfahrungen in den USA - abzusehen, dass auch die Studiengebühren mit erworbenen ECTS-Punkten kombiniert werden können. Studierende würden - so die Idee - dann nicht mehr einen Pauschalbetrag pro Semester bezahlen, sondern ihre Gebühren abhängig von denen ihren Modulen zugerechneten ECTS-Punkten bezahlen. ${ }^{36}$

Aber auch die Leistung von Lehrenden kann an die Kunstwährung ECTS gebunden werden. Bisher wird das Deputat der Lehrenden an den meisten Universitäten nach einem relativ einfachen und bürokratiearmen Schema berechnet. Egal ob ein Lehrender eine Professorin, ein Assistent oder eine Lehrbeauftragte ein Seminar, eine Vorlesung, eine Übung oder ein Kolloquium abhält - jede „Kontaktstunde“ mit den Studierenden wurde ihnen als Lehrdeputatsstunde angerechnet. Durch die ECTS-Punkte hat man jetzt - jedenfalls theoretisch - die Möglichkeit, die Deputate von Lehrenden völlig neu zu bestimmen. Man kann beispielsweise vor- und nachbereitungsintensive Seminarveranstaltungen, in denen Studierende viele ECTS-Punkte erwerben können, bei der Deputatsberechnung für Lehrende höher gewichten als nur mit wenigen ECTS-Punkten gewichtete Übungen, weil man davon ausgehen könnte, dass diese nach Schema X abgehalten werden und von Lehrenden nicht intensiv vor- und nachbereitet werden müssen. Bisher ist dies lediglich theoretische Gedankenspielerei, aber es ist nur eine Frage der Zeit, bis die eine oder andere hochschulpolitische Denkschmiede anfängt, neben den European Credit Transfer

36 Vgl. zur Debatte in den USA Philip Altbach, „Die Messung von Lehr und Lernleistungen: Credit Systeme an US amerikanischen Hochschulen", in: Stefanie Schwarz, Ulrich Teichler (Hrsg.), Credits an deutschen Hochschulen: Kleine Einheiten - große Wirkung, Neuwied, Kriftel 2002, S. 79-89, hier S. 85. 
and Accumulation System auch ein European Teaching Load System (ETLS) zu entwickeln. ${ }^{37}$

Aber auch die Leistungsmessung von Instituten, Fakultäten oder auch ganzer Universitäten kann mit der Kunstwährung ECTS verbunden werden. Im Moment scheint es an vielen Universitäten noch eine abstruse Vorstellung, dass die Gesamtzahl an ECTS-Punkten nicht nur für einzelne Studenten, sondern auch verallgemeinert für ganze Studiengänge einer $\mathrm{Fa}$ kultät oder einer Universität erhoben werden. Bisher klingt es für viele Hochschulangehörige kaum vorstellbar, dass ein Institut irgendwann mal vermeldet, dass im Wintersemester eines Jahres in seinen Veranstaltungen insgesamt 32423 ECTS-Punkte von Studierenden erworben wurden und Universitäten mit Bildungsministerien Leistungsvereinbarungen abschlie-

37 Vgl. für erste solche Gedankenspiele Hener Yorck, "Teaching Points" als neue Planungsgröße für die Lehre? Ein Vorschlag des Centrums für Hochschulentwicklung", in: Forschung \& Lehre, 11/2005, S.600-610. Die Vorstellung von „Teaching Points" als einer „neuen Plangröße“ besteht darin, die „Semesterwochenstunde als Recheneinheit aufzugeben und eine Einheit zu entwickeln, die sich - wie der Credit für die Leistung der Studierenden - am tatsächlichen (sic!) Arbeitsaufwand (workload) der Lehrenden orientiert.“ Dabei müssten ,unterschiedliche Gruppengrößen ebenso berücksichtigt werden wie Vorbereitungs-, Nachbereitungs-, Beratungs- und Prüfungsaufwand." (Yorck a.a.O., S 600). Das klingt in ihren Planungs-, Steuerungs- und Gerechtigkeitsversprechen erst einmal verlockend, ignoriert aber, dass dafür eine ganz eigene Verrechnungs-, Genehmigungsund Kontrollbürokratie aufgebaut werden muss, die - weil der eigentliche Prozess der Lehre in letzter Konsequenz nicht zu kontrollieren ist - und Kontrollen letztlich wiederum nur unterlaufen werden würde. Man kann nur hoffen, dass die Erfinder solcher Modelle sich einmal etwas genauer die bereits bestehenden kleinen Versuche zur Differenzierung von Lehrdeputaten anschauen, z. B. wenn ein Kolloquium nur zur Hälfte als Lehrdeputatsstunde angerechnet wird. Neben einem enormen Planungsaufwand hat dies nur dazu geführt, dass bestimmte Veranstaltungen entweder gar nicht mehr oder nur in der kaschierten Form einer Veranstaltung mit vollem Lehrdeputat angeboten werden. Man kann - einen Buchtitel aus einer dieser Denkschmieden paraphrasierend - von einer von staatlicher Bürokratie ,entfesselten Hochschule" sprechen (vgl. Detlef Müller Böling, Die entfesselte Hochschule, Gütersloh 2000.), die sich dann hoffnungslos in einem Knäuel von detaillierten Richtlinien für Lehre und Studium verheddert, die zwar von den Hochschulen selbst eingeführt wurden und aber von den Denkschmieden mit entsprechender Prosa begleitet wurden. Aber vermutlich würden sich solche Anmerkungen bei den Denkschmieden gar nicht verfangen, weil sie sich gegen Hinweise auf die planwirtschaftlichen Übersteuerungstendenzen ihrer als Effizienzversprechen daherkommenden Reformvorschläge dadurch immunisieren, dass sie Kritik nur als Versuche der „Professoren Untat“ ansehen, ihre Privilegien zu verteidigen. 
ßen, dass in einem Semester ihre Studierenden mindestens 2,5 Mio. ECTS Punkte erwerben, aber durch die IT-Systeme ließen sich diese Zahlen in vielen Hochschulen jetzt schon innerhalb von wenigen Minuten generieren. Erfahrungen mit dem Kreditpunktesystem in den USA und auch erste Erprobungen in europäischen Staaten deuten darauf hin, dass es wohl nur eine Frage der Zeit ist, bis die Kunstwährung auch in die Gesamtsteuerung von Universitäten über Leistungsindikatoren eingebunden wird.

In der Soziologie wird diese Produktion neuer Zahlen durch die Verkopplung einer Zahl mit anderen Zahlen als „Hyperrealität“38 bezeichnet. Zwar mögen von Beobachtern Zweifel an der Logik bei der Verknüpfung zweier Zahlen - z. B. von ECTS-Punkten und Studiengebühren oder von allen an einer Uni erworbenen ECTS-Punkten mit Mittelzuweisungen geäußert werden, aber durch die Fassung von bestimmten Phänomen in die Form von Zahlen entsteht eine Sogwirkung, diese Zahlen mit anderen Zahlen in Beziehung zu setzen und zu verrechnen.

\section{Zu den Sogwirkungen einer Kunstwährung}

Globale Vereinheitlichungen werden häufig als eine Durchsetzung von Standards von oben nach unten beschrieben. Es wird dabei analysiert, wie sich die Entscheidungen von internationalen Organisationen oder internationalen Konferenzen über nationalstaatliche Ratifizierungen bis auf die lokale Ebene durchsetzen. ${ }^{39}$ Oder es wird davon ausgegangen, dass sich weltweit Ideologien in der Form von nahezu quasireligiösen Rationalitätsannahmen über die Funktionsweise von Wirtschaft, Politik, Recht oder Erziehung ausbilden, die dann einen unmittelbaren Anpassungsdruck auf lokale Praktiken ausüben. ${ }^{40}$

38 Hendrik Vollmer, How to Do More With Numbers. Elementary Stakes, Framing, Keying, and the Three dimensional Character of Numerical Signs, Bielefeld 2006, unveröff. Ms.

$39 \mathrm{Vgl}$. für den in diese Richtung argumentierenden regulationstheoretischen Strang z,B. Joachim Hirsch, Materialistische Staatstheorie. Transformationsprozesse des kapitalistischen Staatensystems, Hamburg 2005.

40 Vg1. für den neoinstitutionalistischen Argumentationsstrang z.B. David Strang, John W. Meyer, "Institutional Conditions for Diffusions", in: Theory and Society, Jg. 22, H.4/1993, S. 487-511; John Boli, Frank Lechner, "Globalization and World Culture", in: Neil J. Smelser, Paul B. Baltes (Hrsg.), International Encyclopedia of the Social and Behavioral Sciences, Bd. 9, Amsterdam 2001, S. 6261-6266. 
Der durch die Klammer des Bologna-Prozesses gehaltene Reformprozess scheint sich aber eher von ,unten“ zu bilden. Der zentrale Faktor für die Entstehung und Durchsetzung von Reformmustern scheint dabei in den Experimenten mit der Kunstwährung ECTS zu liegen, den sich die Universitäten, die sich am Reformprozess beteiligen, in der einen oder anderen Form unterwerfen müssen. Man kann hier von der Sogwirkung von Verrechnungseinheiten reden, die bei aller Heterogenität in den Details Entwicklungslinien auf einen gemeinsamen quantitativen Nenner bringen. 
Marianne Kneuer [Hrsg.]

Standortbestimmung Deutschlands: Innere Verfasstheit und internationale Verantwortung 
Die Deutsche Nationalbibliothek verzeichnet diese Publikation in der Deutschen Nationalbibliografie; detaillierte bibliografische Daten sind im Internet über http://dnb.d-nb.de abrufbar.

ISBN 978-3-8487-1196-3 (Print)

ISBN $978-3-8452-5284^{-1}$ (ePDF)

1. Auflage 2015

(C) Nomos Verlagsgesellschaft, Baden-Baden 2015. Printed in Germany. Alle Rechte, auch die des Nachdrucks von Auszügen, der fotomechanischen Wiedergabe und der Übersetzung, vorbehalten. Gedruckt auf alterungsbeständigem Papier. 